\title{
Recovery from Sleep Deprivation Occurs during Propofol Anestbesia
}

\author{
Avery Tung, M.D., * Bernard M. Bergmann, Ph.D., † Stacy Herrera, B.A., $\ddagger$ Dingcai Cao, Ph.D., \\ Wallace B. Mendelson, M.D.\|
}

\begin{abstract}
Background: Some neurophysiologic similarities between sleep and anesthesia suggest that an anesthetized state may reverse effects of sleep deprivation. The effect of anesthesia on sleep homeostasis, however, is unknown. To test the hypothesis that recovery from sleep deprivation occurs during anesthesia, the authors followed $24 \mathrm{~h}$ of sleep deprivation in the rat with a 6-h period of either ad libitum sleep or propofol anesthesia, and compared subsequent sleep characteristics.

Methods: With animal care committee approval, electroencephalographic/electromyographic electrodes and intrajugular cannulae were implanted in 32 rats. After a 7-day recovery and 24-h baseline electroencephalographic/electromyographic recording period, rats were sleep deprived for $24 \mathrm{~h}$ by the diskover-water method. Rats then underwent $6 \mathrm{~h}$ of either propofol anesthesia $(n=16)$ or ad libitum sleep with intralipid administration $(n=16)$, followed by electroencephalographic/ electromyographic monitoring for $72 \mathrm{~h}$.

Results: In control rats, increases above baseline in non-rapid eye movement sleep, rapid eye movement sleep, and non-rapid eye movement delta power persisted for $12 \mathrm{~h}$ after $24 \mathrm{~h}$ of sleep deprivation. Recovery from sleep deprivation in anesthetized rats was similar in timing to that of controls. No delayed rebound effects were observed in either group for $72 \mathrm{~h}$ after deprivation.

Conclusion: These data show that a recovery process similar to that occurring during naturally occurring sleep also takes place during anesthesia and suggest that sleep and anesthesia share common regulatory mechanisms. Such interactions between sleep and anesthesia may allow anesthesiologists to better understand a potentially important source of variability in anesthetic action and raise the possibility that anesthetics may facilitate sleep in environments where sleep deprivation is common.
\end{abstract}

SLEEP deprivation is common in patients in the intensive care unit ${ }^{1}$ and can result in worsened agitation and respi-

This article is accompanied by an Editorial View. Please see: Nelson LE, Franks NP, Maze M: Rested and refreshed after anesthesia? Overlapping neurobiologic mechanisms of sleep and anesthesia. ANESTHESIOLOGY 2004; 100:1341-2.

* Associate Professor of Anesthesia and Critical Care, ‡ Research Technician Department of Anesthesia and Critical Care, † Researcher, Sleep Research Laboratory, $\|$ Professor of Psychiatry and Clinical Pharmacology, Department of Psychiatry, $\mathbb{S}$ Biostatistician, Department of Health Studies, The University of Chicago.

Received from the Departments of Anesthesia and Critical Care and Psychiatry, The University of Chicago, Chicago, Illinois. Submitted for publication September 5, 2003. Accepted for publication January 26, 2004. Supported by the Brain Research Foundation and the Department of Anesthesia and Critical Care, The University of Chicago, Chicago, Illinois, and grant No. K08-GM000697-01A1 from the National Institutes of Health, Bethesda, Maryland. Presented in part at the meeting of the Associated Professional Sleep Societies, Chicago, Illinois, June 7, 2003.

Address reprint requests to Dr. Tung: Department of Anesthesia and Critical Care, The University of Chicago, 5801 South Ellis Avenue, Chicago, Illinois 60637. Address electronic mail to: atung@airway.uchicago.edu. Individual article reprints may be purchased through the Journal Web site, www.anesthesiology.org. ratory, immune, and endocrine system dysfunction. ${ }^{2-4} \mathrm{Al}-$ though naturally occurring sleep readily reverses consequences of sleep deprivation, ${ }^{5}$ such sleep can be difficult to obtain in an intensive care environment. In sleep-deprived critically ill patients, behavioral similarities between levels of sedation approaching general anesthesia and naturally occurring sleep have raised the possibility that the anesthetized state may substitute for sleep and may thus allow recovery from sleep deprivation. ${ }^{6,7}$

Although general anesthesia differs electroencephalographically from naturally occurring sleep, anesthetics may act partly by duplicating activities of brain regions important in initiating or maintaining sleep. ${ }^{8}$ Effects of anesthetics on regional neuronal activity suggest activation of endogenous sleep-promoting pathways. ${ }^{910}$ Sleep deprivation potentiates anesthetic-induced loss of righting reflex, ${ }^{11}$ and anesthetic agents increase sleep when administered into brain regions known to regulate sleep. ${ }^{12}$ In addition, the neurotransmitter adenosine increases sleep, ${ }^{13}$ enhances anesthetic potency, ${ }^{14}$ and delays recovery from halothane anesthesia. ${ }^{15}$ These observations imply that sleep and anesthesia are neurophysiologically related and suggest that anesthesia and sleep may have similar effects on the sleepdeprived state.

During recovery from sleep deprivation, increases in the intensity and amount of non-rapid eye movement (NREM) and rapid eye movement (REM) sleep are observed that are related to the extent of deprivation and represent a homeostatic recovery response. ${ }^{16}$ To test the hypothesis that recovery from sleep deprivation occurs during the anesthetized state, we followed a 24 -h period of sleep deprivation in a rat model with $6 \mathrm{~h}$ of either propofol anesthesia or ad libitum sleep. We then compared NREM and REM sleep characteristics of rats given anesthesia to those of rats allowed to sleep ad lib. If recovery from sleep deprivation were to occur during anesthesia, the duration and degree of the recovery response after emergence should be similar to that observed in controls. If no recovery were to occur, however, the sleepdeprived state should continue throughout the anesthetic. A larger recovery response (manifested as increased NREM and REM intensity and duration) than in controls would then be expected on emergence from anesthesia.

\section{Materials and Methods}

This study was performed with approval from the animal care committee at our institution (Institutional 
Animal Care and Use Committee, University of Chicago, Chicago, Illinois) for the care and use of laboratory animals. Male Sprague-Dawley rats (Harlan Industries, Indianapolis, IN) weighing 250-300 g were anesthetized with intraperitoneal ketamine $(70 \mathrm{mg} / \mathrm{kg})$ and xylazine $(6 \mathrm{mg} / \mathrm{kg}$ ). A silastic PE-10 intravenous catheter (IITC, Woodland Hills, CA) was implanted into the internal jugular vein and tunneled to exit through the neck. During the same surgery, five stainless steel screws (Small Parts Inc., Miami Lakes, FL) were implanted through the skull to serve as dural electroencephalographic electrodes, and two electromyographic electrodes were implanted in the neck musculature. After the surgical procedure, rats recovered for 7 days in a temperature- $\left(21^{\circ}-24^{\circ} \mathrm{C}\right)$ and light-controlled room with ad libitum access to food and water. Lights were turned on at 6:00 $\mathrm{Am}$ and off at 6:00 $\mathrm{PM}$, and the intravenous catheter was flushed every other day with $0.2 \mathrm{ml}$ saline to maintain patency. Including the preoperative phase, rats were adapted to this light cycle for 10-14 days before initiating electrophysiologic monitoring.

After the 7-day recovery period, rats were acclimated to the disk-over-water sleep deprivation apparatus for $24 \mathrm{~h}$ in preparation for the deprivation protocol. In this paradigm, rats are placed on a 45-cm-diameter disk suspended horizontally over a pan of water with continuous computerized electroencephalographic/electromyographic monitoring. When sleep onset is detected, the computer rotates the disk at a rate of 3 revolutions/min, causing the rat to wake up and walk to avoid falling into the water. When the rat awakens, rotation stops. This method has been previously validated as able to produce near-total sleep deprivation without excessive physical exertion. ${ }^{17}$

During the initial 24-h acclimation period, rats were placed in the disk-over-water apparatus with a platform over the wheel to eliminate the water hazard and to allow ad libitum activity. After acclimation, electroencephalographic/electromyographic recordings were obtained for a 24-h period beginning and ending at 12:00 noon to establish baseline values. Immediately after the baseline period, the platform was removed and rats underwent a 24-h period of total sleep deprivation. Temperature and lighting conditions in the apparatus during baseline and deprivation were the same as for adaptation $\left(21^{\circ}-24^{\circ} \mathrm{C}\right.$ and lights on from 6:00 AM to 6:00 PM).

When the sleep-deprivation period ended, the platform was replaced, and 16 rats were anesthetized with a continuous infusion of propofol (Zeneca Pharmaceuticals, Wilmington, DE) diluted to $5 \mathrm{mg} / \mathrm{ml}$ with $0.9 \%$ saline and administered via syringe pump (Baxter AS50; Baxter Healthcare Corp., Round Lake, IL). Sedation was begun at a propofol dose of $500 \mu \mathrm{g} \cdot \mathrm{kg}^{-1} \cdot \mathrm{min}^{-1}$ and continued until the righting reflex was lost and the rat was able to tolerate clip-type pulse oximetry without moving. Sedation was then titrated downward at 5-min intervals to the lowest level required to maintain loss of righting reflex and tolerance of pulse oximetry and to prevent spontaneous movement with gentle prodding. Rats were allowed to breathe spontaneously, rectal temperature was maintained higher than $36^{\circ} \mathrm{C}$ via heat lamp, and continuous pulse oximetry (Ohmeda Biox 3740; Ohmeda, Madison, WI) was used to verify oxygen saturation greater than $90 \%$. Vital signs, infusion rates, and rat behavior were continuously monitored and recorded every $15 \mathrm{~min}$. The infusion was continued for a total of $5.5 \mathrm{~h}$ (12:00 PM-5:30 PM). Control rats for which ad libitum sleep was allowed $(\mathrm{n}=16)$ underwent the same protocol but received an infusion of $5 \%$ intralipid (Baxter Healthcare, Deerfield, IL) at $1 \mathrm{ml} / \mathrm{h}$, a rate equal to the average $\mathrm{ml} / \mathrm{h}$ rate for rats receiving propofol. At 5:30 PM, all infusions were discontinued, and the electroencephalogram/electromyogram was monitored continuously in both groups for $72 \mathrm{~h}$ to determine the time course and characteristics of NREM and REM recovery sleep.

Electroencephalographic and electromyographic data for all rats were recorded on a Grass model 78 polygraph (Grass-Telefactor, West Warwick, RI) with a paper speed of $10 \mathrm{~mm} / \mathrm{s}$ and also relayed to a computer for digital recording and spectral analysis. Electroencephalographic and electromyographic data were divided into 30-s epochs and were scored as waking, NREM, or REM sleep using an automated scoring system previously validated against visual and behavioral methods. ${ }^{17,18}$ Portions of the electroencephalogram/electromyogram were also scored visually to verify the reliability of the automated system. Definitions of sleep stages have been presented in detail previously. ${ }^{18}$

In addition to sleep staging, spectral analysis was performed on the midline electroencephalogram. After amplification and filtering $(0.5-18 \mathrm{~Hz}[3 \mathrm{~dB}$ points, $12 \mathrm{~dB} / \mathrm{oct}]$ ), the electroencephalogram was sampled at $64 \mathrm{~Hz}$. Using a 4-s window with a Hanning window vector to minimize artifact at the borders of each window, six equally-spaced fast Fourier transforms were performed for each 30-s epoch to calculate the frequency distribution of electroencephalographic power. Low-frequency delta $(2-4 \mathrm{~Hz})$ power was extracted for each window, averaged over the epoch, and divided by bandwidth to obtain power density.

At the conclusion of all electroencephalographic/electromyographic recording, all rats were killed by intraperitoneal injection of $300 \mathrm{mg} / \mathrm{kg}$ pentobarbital.

\section{Statistical Analysis}

All statistical analyses were performed using SAS version 8 (SAS Institute, Cary, NC). NREM sleep stage data were collected for each rat, averaged in $3 \mathrm{~h}$ blocks, and expressed as a percent of recording time. Because REM sleep comprised less than $6 \%$ of total recording time and was absent during much of the active phase, REM sleep data (expressed as number of epochs) was averaged into 


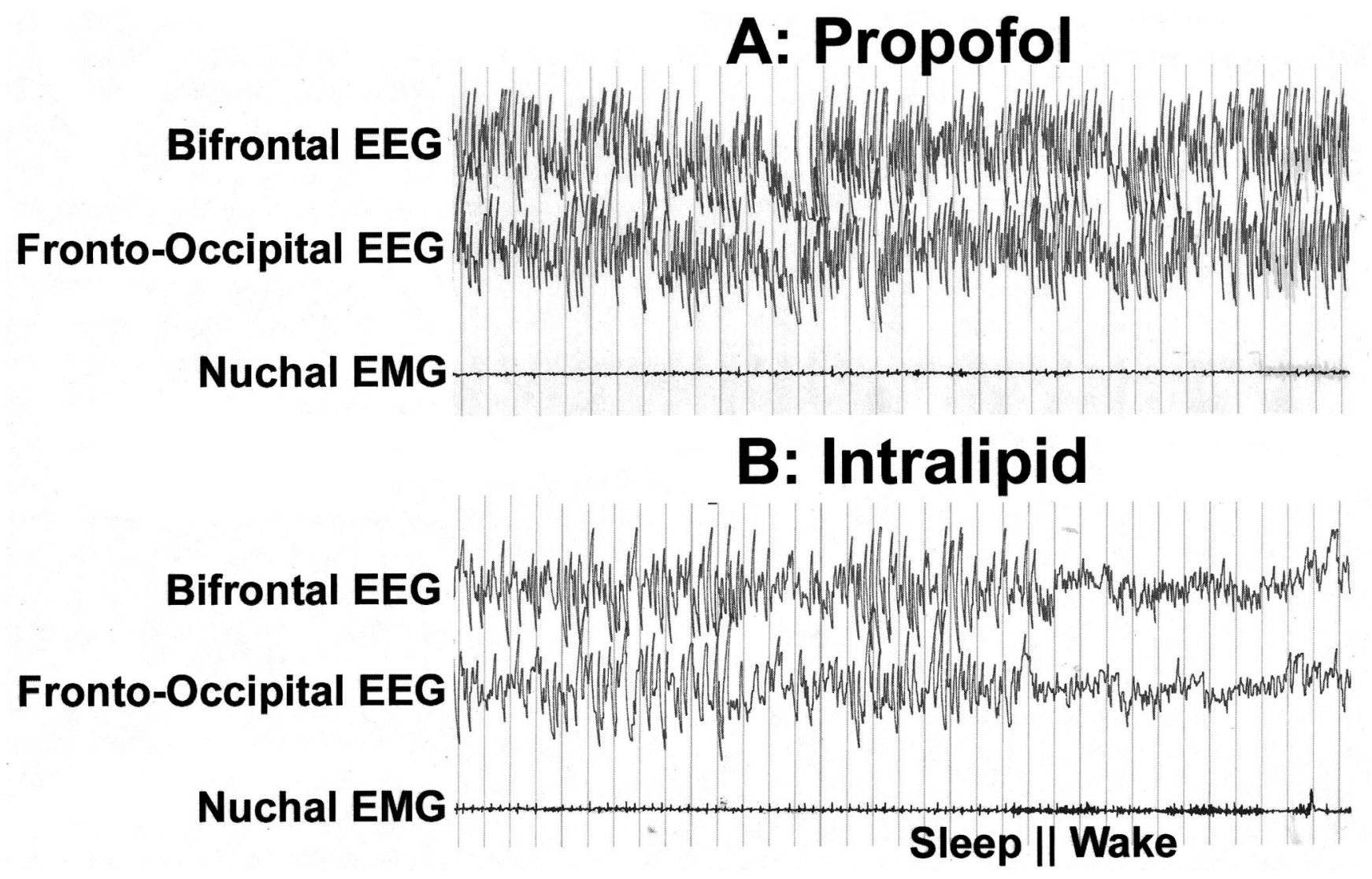

Fig. 1. Sample electroencephalographic/electromyographic recordings from rats anesthetized with propofol $(A)$ and control rats given intralipid (B). Paper speed was $10 \mathrm{~mm} / \mathrm{s}$. In intralipid rat recording, sleep-to-wake transition is marked. EEG = electroencephalogram; EMG = electromyogram.

larger, 6-h blocks to ensure that all blocks contained some REM sleep. To correct for differences in mean delta power density due to variability in electrode placement or skull shape, delta power during epochs scored as NREM sleep was normalized by dividing the NREM values for each rat for each block by the mean delta power density during REM sleep for the same block. To prevent division by zero in blocks with no REM sleep, delta power was thus averaged into 6-h blocks. This approach has previously been reported to minimize within-group variance in rodents. ${ }^{19}$

Baseline sleep for propofol and intralipid groups were compared using a repeated-measures analysis of variance (ANOVA). For each group, comparisons between postdeprivation recovery and baseline sleep were also performed using a repeated-measures ANOVA.

To determine the magnitude and extent of postdeprivation rebound sleep, each 3-h block of postdeprivation recovery sleep was first normalized by subtracting baseline sleep at the same time point. This step served to remove the normal circadian variation in sleep with time. Each 3-h block of normalized postdeprivation sleep was then compared to 0 using a one-sided $t$ test with Bonferroni adjustments for multiple comparisons.

Between-groups comparisons of postdeprivation sleep characteristics was performed by subtracting baseline values from postdeprivation sleep in both groups and comparing the resulting differences using a block-byblock repeated-measures ANOVA.

\section{Results}

For both intralipid and propofol groups, average weights ( $266.1 \pm 19 v$ s. $279.8 \pm 22 \mathrm{~g})$, ages $(71.3 \pm 6.3$ vs. $70 \pm 6.1$ days), and degrees of sleep deprivation $(92.9 \pm 6.3$ vs. $90.5 \pm 9.2 \%$ wake/24 h) were similar. During deprivation, there was no significant difference in disk rotation between rats in the intralipid (18.3 \pm 9.4\%/24-h period) and propofol (15.4 $\pm 13 \%)$ groups. The average infusion rate for rats sedated with propofol over the 6-h period was $297 \pm 38 \mu \mathrm{g} \cdot \mathrm{kg}^{-1} \cdot \mathrm{min}^{-1}$, corresponding to a total infusion volume of $5.95 \pm$ $0.6 \mathrm{ml}$ over $6 \mathrm{~h}$. Control rats received a total infusion volume of $6 \mathrm{ml}$ over $6 \mathrm{~h}(1 \mathrm{ml} / \mathrm{h})$. Visual inspection of the electroencephalogram during propofol anesthesia revealed a continuous, high-amplitude pattern without noticeable NREM or REM stages for the entire 6-h period (fig. 1). During the 6-h intervention period, sleep in control rats (NREM + REM) totaled $4.27 \pm 0.33 \mathrm{~h}$. Rats anesthetized with propofol took less than $10 \mathrm{~min}$ to reach their behavioral endpoint, defined as a loss of 


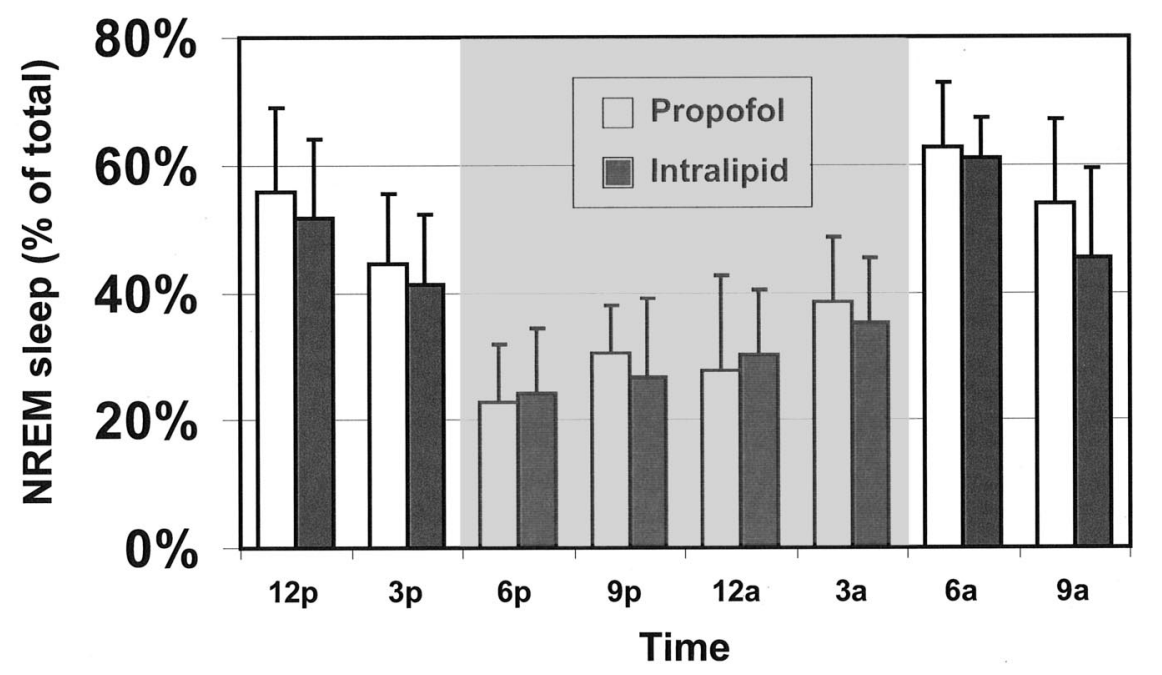

Fig. 2. Non-rapid eye movement (NREM) sleep frequency during baseline recording. NREM sleep was scored in 30-s epochs, collected and averaged in $3-\mathrm{h}$ blocks, and expressed as percent of total block time \pm SD. Shaded areas depict dark phase (6:00 PM-6:00 AM) recording. $\mathrm{AM}$ and $\mathrm{PM}$ are denoted as $a$ and $p$, respectively.

righting reflex and tolerance of clip-style pulse oximetry. The average time from discontinuing the infusion to first movement was $30 \pm 18 \mathrm{~min}$.

\section{NREM Sleep}

Baseline values for NREM sleep in control rats given intralipid and anesthetized rats given propofol were similar (fig. 2). Both groups demonstrated normal circadian variability (less sleep during the lights-off period between 6:00 Pм and 6:00 Am) and appropriate synchronization to the experimental lighting cycle. In controls, NREM sleep after deprivation was significantly increased above baseline values (obtained at the same time point) for $12 \mathrm{~h}$ before returning to baseline (fig. 3). Specifically, NREM sleep was increased above baseline by (mean \pm SD) $19.3 \pm 3.0 \%$ in the first 3 -h block (12:00 PM-3:00 PM, $\mathrm{t}(15)=6.38, P<0.001), 18.1 \pm 8.3 \%$ in the second block (3:00 Pм-6:00 Pм, t(15) $=8.76, P<0.001), 14.0 \pm$ $10.6 \%$ in the third block (6:00 РM-9:00 AM, $\mathrm{t}(15)=5.30$, $P<0.001)$, and $9.3 \pm 11.7 \%$ in the fourth block (9:00 AM-12:00 Aм, t(15) $=3.17, P=0.025)$. NREM sleep returned to baseline levels during the fifth block and did not differ from baseline for the remainder of the 72-h recording period. In particular, no delayed rebound was observed.

In rats given propofol, conventional scoring of the electroencephalogram/electromyogram during the 6-h anesthetic was not performed. No comparisons to baseline were therefore made for the first two blocks after deprivation. After emergence, NREM sleep was increased by $22.3 \pm 18.0 \%$ in the first 3 -h block only $(\mathrm{t}(15)=4.45, P<0.002$; fig. 3$)$. NREM sleep returned to baseline during the second 3 -h block after emergence from anesthesia and was not different from baseline for the subsequent 72 -h recording period. The overall duration of increased sleep after deprivation (including the 6-h anesthetic) was thus $9 \mathrm{~h}$. As with control rats, no delayed increase in postdeprivation NREM sleep was observed.

When NREM sleep had returned to baseline levels in both groups, no delayed increases were observed, and circadian variation appeared normal for the duration of the
$50 \%$

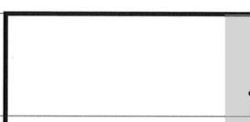

$40 \%$

$30 \%$

$20 \%$

$10 \%$

$0 \%$
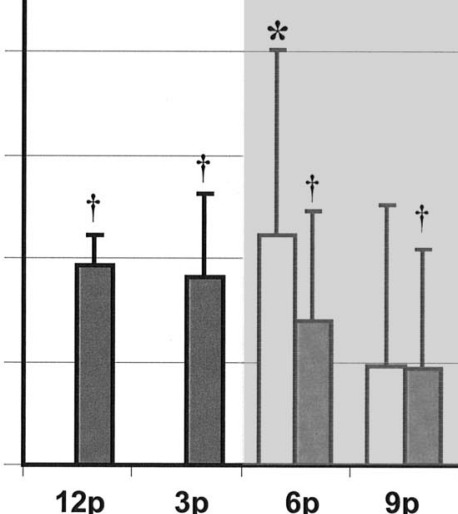

$12 p$

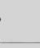

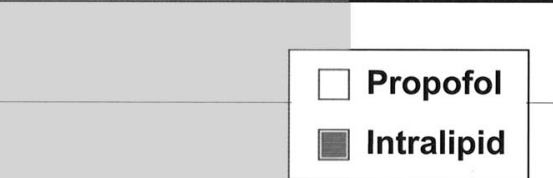

lipid
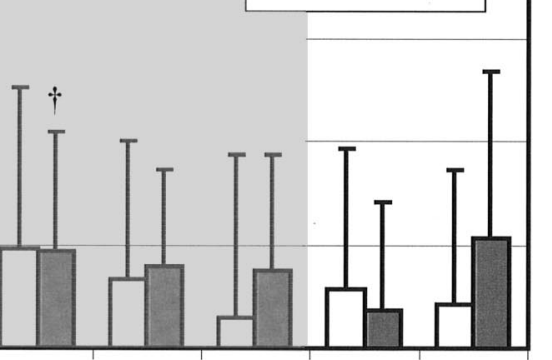

$3 p$
$6 p$
$9 p$

Time
Fig. 3. Rebound non-rapid eye movement (NREM) sleep after deprivation for propofol and control animals. Values were calculated by collecting and averaging NREM sleep in 3-h blocks before and after deprivation, subtracting postdeprivation NREM sleep from baseline values obtained at the same circadian time point and expressed as mean change in absolute percentage from baseline values \pm SD. Note that NREM behavior was not plotted for propofol rats during anesthesia. Shaded areas depict dark phase (6:00 PM-6:00 AM) recording. *,$+P<0.05$ when compared with baseline values for propofol and intralipid groups, respectively. AM and PM are denoted as $a$ and $p$, respectively. 
Fig. 4. Rapid eye movement (REM) sleep at baseline and after deprivation. REM sleep was scored in 30-s epochs, collected in 6-h blocks, and expressed as mean number of epochs/time block \pm SD. Note that REM behavior was not plotted for propofol rats during anesthesia. Shaded areas depict dark phase (6:00 PM-6:00 AM) recording. *, $† P<0.05$ when compared with baseline values for propofol and intralipid groups, respectively. AM and $\mathrm{PM}$ are denoted as $a$ and $p$, respectively.

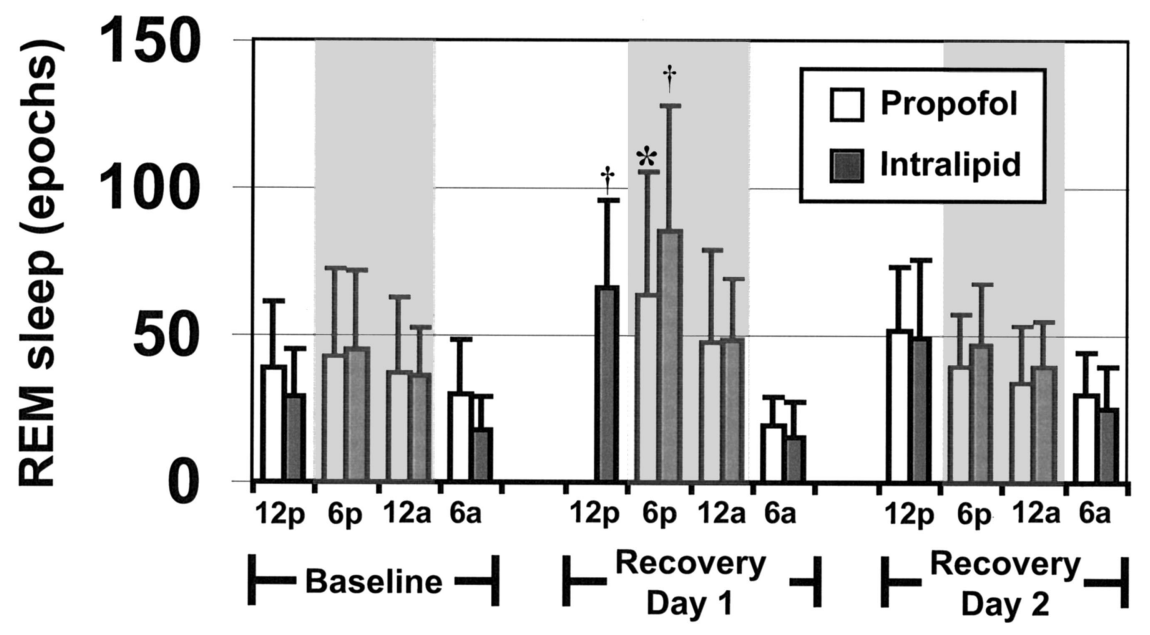

72-h recording period. When postdeprivation NREM sleep was directly compared between propofol and intralipid groups, a repeated-measures ANOVA showed more NREM sleep in controls than in anesthetized rats during only one 3-h block after deprivation, with no other differences between the two groups for the entire recording period. In no postdeprivation block did NREM sleep for propofol rats significantly exceed that of controls.

\section{REM Sleep}

As with baseline values for NREM sleep, baseline values for REM sleep in propofol and intralipid groups were similar by repeated-measures ANOVA. In both groups, a characteristic circadian pattern with REM sleep greatest in the second block (6:00 PM-12:00 AM) was observed (fig. 4). In control rats, sleep deprivation significantly increased REM sleep for the first two 6-h blocks before returning to baseline $(37.4 \pm 27.6$ epochs, $\mathrm{t}(15)=5.41$, $P<0.001$ for the first block, 12:00 PM- 6:00 PM; 40.2 \pm 40.1 epochs, t(15) $=3.95, P<0.001$ for the second block, 6:00 PM-12:00 AM) (fig. 4). The duration of REM sleep rebound was thus $12 \mathrm{~h}$. After deprivation, REM sleep for each recovery day was greatest during the first block (12:00 PM6:00 PM), suggesting residual sleep deprivation.
In rats given propofol, REM sleep was not evaluated during the 6-h propofol anesthetic. After emergence, REM sleep was increased for 6 h (6:00 PM-12:00 AM, 20.8 \pm $40.1, \mathrm{t}(15)=2.45, P=0.05)$ before returning to baseline. The REM rebound response in propofol rats was thus complete $12 \mathrm{~h}$ after the end of deprivation. As with control rats, rats given propofol demonstrated the same altered daily pattern of REM sleep after deprivation, suggesting a similar degree of residual sleep deprivation (fig. 4).

When postdeprivation REM sleep was normalized in both groups by subtracting baseline values, repeatedmeasures ANOVA showed slightly higher REM sleep in control rats during the fourth block after deprivation but no other differences between intralipid and propofol rats. At no time after deprivation was REM sleep in propofol rats increased above that in controls.

\section{NREM Delta Power}

During baseline monitoring, delta power did not differ significantly between anesthetized and control animals. In both groups, baseline delta power was highest during the third block (12:00 AM-6:00 AM). In control rats, sleep deprivation significantly increased NREM delta power in the first 6-h block on the first recovery day $(1.61 \pm 1.4$,
Fig. 5. Delta power during non-rapid eye movement (NREM) sleep at baseline and after deprivation. Delta power was calculated during epochs scored as NREM sleep, collected in 6-h blocks, and normalized by dividing by average REM delta power for the same period. Values were expressed as mean \pm SD. Note that delta behavior was not plotted for propofol rats during anesthesia. Hatched bars indicate the intralipid group; open bars indicate the propofol group. Shaded areas depict dark phase (6:00 PM-6:00 AM) recording. *, $\dagger \boldsymbol{P}<0.05$ when compared with baseline values for propofol and intralipid groups, respectively. AM and PM are denoted as $a$ and $p$, respectively.

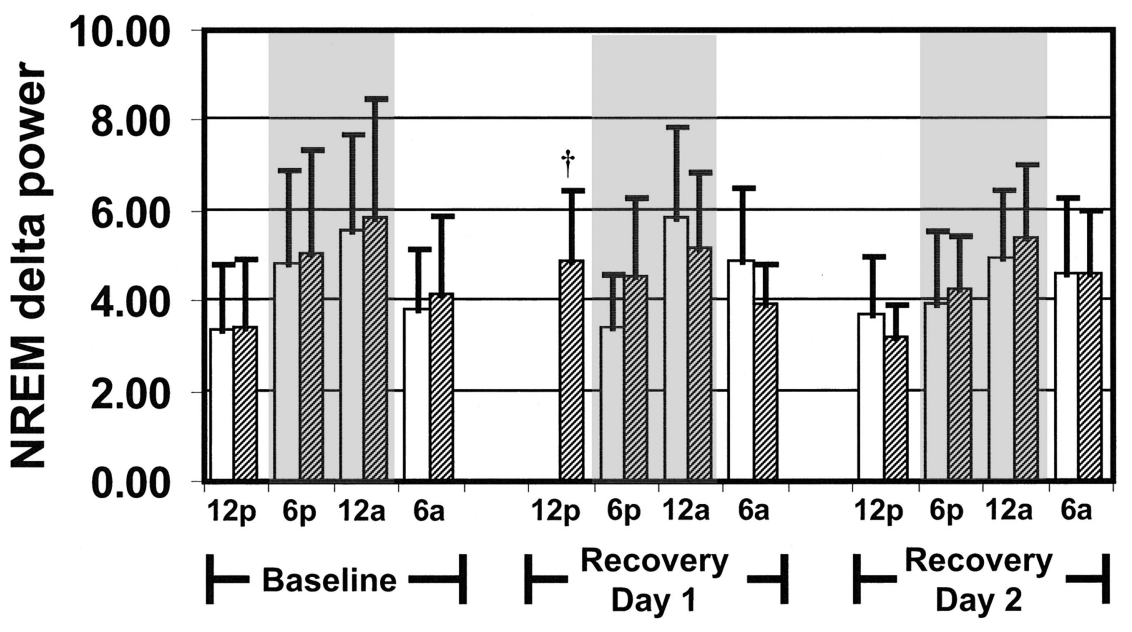


$\mathrm{t}(15)=3.61, P<0.005)$. NREM delta subsequently returned to baseline during the second block and was indistinguishable from baseline for the duration of the 72-h monitoring period (fig. 5).

In rats anesthetized with propofol, delta power was not calculated during the anesthetic. Absolute delta power, however, was negligible for the entire anesthetic, a finding consistent with propofol anesthesia in rats. ${ }^{20}$ After emergence, delta power was not increased above baseline in any block for the duration of the $72-\mathrm{h}$ recording period.

When postdeprivation delta power was normalized to baseline and compared between groups, no differences were found. Absolute theta power, characteristically increased during REM sleep and some waking periods, ${ }^{18}$ was negligible in rats receiving propofol.

\section{Discussion}

We found that after $24 \mathrm{~h}$ of sleep deprivation, recovery sleep behavior in rats for which $6 \mathrm{~h}$ of ad libitum sleep was allowed was no different from that of rats subjected to a 6-h propofol anesthetic. After emergence from propofol anesthesia, increases in NREM sleep, REM sleep, and delta power suggesting recovery from sleep deprivation persisted for 9,12 , and $6 \mathrm{~h}$, respectively. These increases were equal to or slightly less than those for control rats for which ad libitum recovery was allowed. In addition, when sleep had returned to baseline in rats given propofol, no delayed increase in any form of sleep was seen for the duration of the 72-h monitoring period.

In principle, a period of anesthesia might modify the homeostatic regulation of sleep debt in three ways. For example, general anesthesia might be a permissive state that allows normal sleep homeostatic processes to occur. An anesthetized organism would thus repay sleep debt built up during previous wakefulness and emerge less sleep-deprived after an anesthetic than before. Alternatively, anesthesia might progressively increase sleep debt in a fashion similar to wakefulness. Prolonged anesthetics would then induce a sleep-deprived state. Finally, anesthesia might represent a state unlike either sleep or waking, in which sleep debt neither accumulates nor dissipates. Organisms emerging from anesthesia would then have the same degree of sleep deprivation as when they were initially anesthetized.

Under normal conditions, robust sleep homeostatic mechanisms act to preserve adequate sleep after sleep deprivation. Even mild sleep loss increases the propensity to sleep, ${ }^{5}$ and sustained deprivation can cause the organism to sleep when doing so would be life-threaten-

\footnotetext{
\# National Heart, Lung and Blood Institute/National Center for Sleep Disorder Research: Drowsy driving and automobile crashes. Available at: http://www.nhlbi.nih.gov/health/prof/sleep/drsy_drv.htm. Accessed December 31, 2003.
}

ing.\# Recovery from sleep deprivation is characterized by rebound increases in NREM and REM sleep and changes in the power spectrum of NREM sleep that persist until homeostatic mechanisms have been satisfied. ${ }^{16}$

Our findings are consistent with the presence of an active sleep homeostatic process during anesthesia with propofol. If the anesthetized state had resembled wakefulness in its effect on sleep homeostasis, additional sleep "debt" would have accrued throughout the 6-h anesthetic. This increased debt, combined with a delay in the initiation of recovery until after emergence, would have resulted in a greater amount and intensity of NREM and REM sleep when compared with controls, a higher delta power during NREM sleep, and a corresponding delay in the return of NREM and REM sleep to baseline. If sleep debt had remained static during anesthesia, recovery from deprivation would also have been delayed until after the anesthetic and would have manifested as higher levels of sleep and NREM delta power compared with controls and a longer duration of recovery. Our observation that rats anesthetized with propofol had recovery characteristics nearly identical to rats for which unrestricted sleep was allowed strongly suggests that a recovery process occurred during the 6-h anesthetic and implies that anesthesia with propofol affects sleep homeostasis in a fashion similar to naturally occurring sleep.

Although the mechanisms by which sleep reverses behavioral manifestations of sleep deprivation are poorly understood, plausible links between anesthesia and sleep regulatory mechanisms support an ability of the brain to recover from sleep deprivation during anesthesia. Sleep deprivation increases extracellular adenosine concentrations in the basal forebrain of rats and cats, a site known to modulate central nervous system arousal. ${ }^{13}$ Evidence that administration of adenosine reuptake inhibitors into the basal forebrain increases sleep ${ }^{21}$ and that adenosine delays recovery from halothane anesthesia $^{15}$ suggests that extracellular adenosine concentrations plays a role in control mechanisms for both sleep and anesthesia. Increases in basal forebrain adenosine may potentiate anesthetic action, for example, and reduced metabolic demands during anesthesia may allow adenosine concentrations built up during wakefulness to dissipate. Alternatively, anesthetic-induced changes in brain activity may directly decrease adenosine release.

Neurophysiologic similarities between anesthesia and sleep may also allow the anesthetized state to reverse behavioral effects of sleep deprivation. Positron electron tomography/metabolic scanning ${ }^{9}$ and microelectrode recordings of thalamic relay neuronal activity ${ }^{22}$ both demonstrate reductions in thalamic activity during anesthesia, an important characteristic of naturally occurring sleep. ${ }^{23}$ Histologic studies suggest that dexmedetomidine anesthesia increases activity in the ventrolateral preoptic nucleus and reduces activity in the locus ceruleus ${ }^{10}$ in a fashion similar to sleep. Although the mo- 
lecular mechanisms governing sleep homeostasis are unknown, some feature of brain activity during anesthesia may duplicate the aspect of naturally occurring sleep that modulates homeostatic control of sleep.

We did not test other anesthetic agents for two reasons. Because our central finding was that no difference existed between anesthetized and control groups, we believed that to include another group would have weakened the statistical finding of no difference. In addition, other anesthetics, such as isoflurane, may have had delayed effects on sleep patterns, preventing us from interpreting postdeprivation behavior. We also targeted a single, behaviorally defined endpoint. Because sleep deprivation can affect anesthetic potency, ${ }^{11}$ fixed doses of anesthetic would have resulted in changes in the depth of anesthesia as the degree of deprivation changed over time. We therefore titrated our anesthetic to loss of righting reflex and tolerance of clip-style pulse oximetry to maximize behavioral similarities to sleep. Although a dose-response curve involving a different behavioral endpoint (such as electroencephalographic silence) was possible, cardiorespiratory depression would have necessitated intubation and mechanical ventilation for the duration of the anesthetic.

Delayed, propofol-induced effects on sleep may also have altered the interpretation of our results. In humans, the combination of inhaled anesthetics and surgery results in initial suppression of REM sleep, followed by a rebound increase on the second or third postoperative day. ${ }^{24}$ We thought it unlikely in our study that a delayed effect of propofol anesthesia suppressed subsequent sleep and obscured detection of a recovery response. No delayed rebound suggestive of an initial suppressive effect was seen in anesthetized rats, and block-by-block comparisons between baseline and postanesthesia sleep after the end of recovery revealed no suppressive effect in any sleep measure. It is possible that the shorter recovery duration in anesthetized animals actually indicated a more efficient recovery process during anesthesia than during ad libitum conditions. This possibility might plausibly be explained by differences in the time spent in the unresponsive state $(6 \mathrm{~h}$ for anesthetized rats $v s .4 .27 \mathrm{~h}$ for controls).

Finally, lighting conditions during our study deserve mention. Rats are nocturnal animals, normally asleep during daylight hours. ${ }^{25}$ At baseline, rats in both groups demonstrated appropriately less sleep during the dark period. In our study design, the 6-h intervention occurred during the light phase (12:00 PM-6:00 PM), with the next $12 \mathrm{~h}$ spent in darkness (6:00 PM-6:00 AM). We chose this strategy because recovery during the light phase is subject to a "ceiling effect" on total sleep, ${ }^{26}$ which may have limited the size of the rebound we observed. Locating the infusion period in the last $6 \mathrm{~h}$ of the light phase thus allowed us to contrast high lightphase recovery sleep levels in controls to near-zero overt sleep in anesthetized animals and examine recovery dur- ing darkness when between-groups comparisons would be optimally sensitive to differences in recovery sleep. Although previous studies in our laboratory have examined recovery from sleep deprivation under constant lighting conditions, ${ }^{25,26}$ removing such a circadian cue introduces the possibility of circadian drift over time. We therefore chose to monitor recovery under baseline, 12:12-h lighting conditions to minimize this possibility. Because propofol and control rat recovery data were compared at the same point in their diurnal cycle and time after deprivation, phase shifts and diurnal variation were unlikely to affect our results.

In summary, we report that after a 24 -h period of sleep deprivation, rats anesthetized for $6 \mathrm{~h}$ with propofol recovered to the same degree as rats allowed $6 \mathrm{~h}$ of ad libitum sleep. This observation suggests that sleep and anesthesia may share common control mechanisms and raises the possibility that understanding anesthetic effects on known correlates of sleep homeostasis may facilitate knowledge regarding effects and consequences of sleep deprivation. Clinically, such interactions between sleep and anesthesia may allow anesthesiologists to better understand how sleep deprivation and anesthesia interact and may potentially allow anesthetics to facilitate sleep in environments where sleep deprivation is common.

The authors thank Martin J. Szafran, B.A. (Department of Anesthesia and Critical Care, The University of Chicago, Chicago, Illinois), for his valuable technical assistance with this study.

\section{References}

1. Cooper AB, Thornley KS, Young GB, Slutsky AS, Stewart TE, Hanly PJ: Sleep in critically ill patients requiring mechanical ventilation. Chest 2000; 117:809-18

2. Chen HI, Tang YR: Sleep loss impairs inspiratory muscle endurance. Am Rev Respir Dis 1989; 140:907-9

3. Irwin M: Effects of sleep and sleep loss on immunity and cytokines. Brain Behav Immun 2002; 16:503-12

4. Spiegel K, Leproult R, Van Cauter E: Impact of sleep debt on metabolic and endocrine function. Lancet 1999; 354:1435-9

5. Bonnet MH: Sleep deprivation, Principles and Practice of Sleep Medicine, 2nd edition. Edited by Kryger MH, Roth T, Dement W. Philadelphia, Saunders, 1994, pp 50-67

6. Manthous CA, Schmidt GA, Hall JB: Liberation from mechanical ventilation: A decade of progress. Chest 1998; 114:886-901

7. Treggiari-Venzi M, Borgeat A, Fuchs-Buder T, Gachoud JP, Suter PM: Overnight sedation with midazolam or propofol in the ICU: Effects on sleep quality, anxiety and depression. Intensive Care Med 1996; 22:1186-90

8. Lydic R, Biebuyck JF: Sleep neurobiology: Relevance for mechanistic studies of anaesthesia. Br J Anaesth 1994; 72:506-8

9. Alkire MT, Pomfrett CJ, Haier RJ, Gianzero MV, Chan CM, Jacobsen BP, Fallon JH: Functional brain imaging during anesthesia in humans: Effects of halothane on global and regional cerebral glucose metabolism. ANESTHESIOLOGY 1999; 90:701-9

10. Nelson LE, Lu J, Guo T, Saper CB, Franks NP, Maze M: The $\alpha_{2}$-adrenoceptor agonist dexmedetomidine converges on an endogenous sleep-promoting pathway to exert its sedative effects. ANesTHESIOLOGY 2003; 98:428-36

11. Tung A, Szafran MJ, Bluhm B, Mendelson WB: Sleep deprivation potentiates the onset and duration of loss of righting reflex induced by propofol and isoflurane. ANESTHESIOLOGY 2002; 97:906-11

12. Tung A, Bluhm B, Mendelson WB: The hypnotic effect of propofol in the medial preoptic area of the rat. Life Sci 2001; 69:855-62

13. Porkka-Heiskanen T, Strecker RE, Thakkar M, Bjorkum AA, Greene RW, McCarley RW: Adenosine: A mediator of the sleep-inducing effects of prolonged wakefulness. Science 1997; 276:1265-8

14. Kaputlu I, Sadan G, Ozdem S: Exogenous adenosine potentiates hypnosis induced by intravenous anaesthetics. Anaesthesia 1998; 53:496-500 
15. Tanase D, Baghdoyan HA, Lydic R: Dialysis delivery of an adenosine A1 receptor agonist to the pontine reticular formation decreases acetylcholine release and increases anesthesia recovery time. ANESTHESIOLOGY 2003; 98:912-20

16. Borbely AA, Tobler I, Hanagasioglu M: Effect of sleep deprivation on sleep and EEG power spectra in the rat. Behav Brain Res 1984; 14:171-82

17. Bergmann BM, Kushida CA, Everson CA, Gilliland MA, Obermeyer W, Rechtschaffen A: Sleep deprivation in the rat: II. Methodology. Sleep 1989; 12:5-12

18. Bergmann BM, Winter JB, Rosenberg RS, Rechtschaffen A: NREM sleep with low-voltage EEG in the rat. Sleep 1987; 10:1-11

19. Naylor E, Bergmann BM, Krauski K, Zee PC, Takahashi JS, Vitaterna MH, Turek FW: The circadian clock mutation alters sleep homeostasis in the mouse. J Neurosci 20000; 20:8138-43

20. Tan PPC, Shyr MH, Yang CH, Kuo TBJ, Pan WHT, Chan SHH. Power spectral analysis of the electroencephalographic and hemodynamic correlates of propofol anesthesia in the rat: Intravenous infusion. Neurosci Lett 1993; 160: 205-8

21. Strecker RE, Morairty S, Thakkar MM, Porkka-Heiskanen T, Basheer R,
Dauphin LJ, Rainnie DG, Portas CM, Greene RW, McCarley RW: Adenosinergic modulation of basal forebrain and preoptic/anterior hypothalamic neuronal activity in the control of behavioral state. Behav Brain Res 2000; 115:183-204

22. Vahle-Hinz C, Detsch O, Siemers M, Kochs E, Bromm B: Local GABA(A) receptor blockade reverses isoflurane's suppressive effects on thalamic neurons in vivo. Anesth Analg 2001; 92:1578-84

23. Jones BE: Basic mechanisms of sleep-wake states, Principles and Practice of Sleep Medicine, 2nd edition. Edited by Kryger MH, Roth T, Dement W. Philadelphia, Saunders, 1994, pp 145-62

24. Knill RL, Moote CA, Skinner MI, Rose EA: Anesthesia with abdominal surgery leads to intense REM sleep during the first postoperative week. ANESTHESIOLOGY 1990; 73:52-61

25. Bergmann BM, Mistlberger RE, Rechtschaffen A: Period-amplitude analysis of rat electroencephalogram: Stage and diurnal variations and effects of suprachiasmatic nuclei lesions. Sleep 1987; 10:523-36

26. Mistlberger RE, Bergmann BM, Waldenar W, Rechtschaffen A: Recovery sleep following sleep deprivation in intact and suprachiasmatic nuclei-lesioned rats. Sleep $1983 ; 6: 217-33$ 